The

Minimum

Description

Length

Principle 

The

\section{Minimum \\ Description \\ Length \\ Principle}

Peter D. Grünwald

The MIT Press

Cambridge, Massachusetts

London, England 
(C) 2007 Massachusetts Institute of Technology

All rights reserved. No part of this book may be reproduced in any form by any electronic or mechanical means (including photocopying, recording, or information storage and retrieval) without permission in writing from the publisher.

Typeset in Palatino by the author using $\mathrm{LT}_{\mathrm{E}} \mathrm{X} 2 \varepsilon$ with C. Manning's fbook. cls and statnlpbook. sty macros.

Printed and bound in the United States of America.

Library of Congress Cataloging-in-Publication Information

Grünwald, Peter D.

The minimum description length principle / Peter D. Grünwald.

p. cm.-(Adaptive computation and machine learning)

Includes bibliographical references and index.

ISBN-13: 978-0-262-07281-6 (alk. paper)

1. Minimum description length (Information theory) I. Title

QA276.9G78 2007

003'.54-dc22

2006046646

10987654321 
To my father 



\title{
Brief Contents
}

\author{
I Introductory Material $\mathbf{1}$ \\ 1 Learning, Regularity, and Compression 3 \\ 2 Probabilistic and Statistical Preliminaries 41 \\ 3 Information-Theoretic Preliminaries 79 \\ 4 Information-Theoretic Properties of Statistical Models 109 \\ 5 Crude Two-Part Code MDL 131 \\ II Universal Coding 165 \\ 6 Universal Coding with Countable Models 171 \\ 7 Parametric Models: Normalized Maximum Likelihood 207 \\ 8 Parametric Models: Bayes 231 \\ 9 Parametric Models: Prequential Plug-in 257 \\ 10 Parametric Models: Two-Part 271 \\ 11 NML With Infinite Complexity 295 \\ 12 Linear Regression 335 \\ 13 Beyond Parametrics 369
}

III Refined MDL 403

14 MDL Model Selection 409

15 MDL Prediction and Estimation 459

16 MDL Consistency and Convergence 501

17 MDL in Context 523 
IV Additional Background 597

18 The Exponential or "Maximum Entropy" Families 599

19 Information-Theoretic Properties of Exponential Families 


\section{Contents}

List of Figures $\quad$ ix
Series Foreword $\quad x x i$
Foreword xxiii
Preface xxv

\section{Introductory Material 1}

1 Learning, Regularity, and Compression 3

1.1 Regularity and Learning 4

1.2 Regularity and Compression 4

1.3 Solomonoff's Breakthrough - Kolmogorov Complexity 8

1.4 Making the Idea Applicable 10

1.5 Crude MDL, Refined MDL and Universal Coding 12

1.5.1 From Crude to Refined MDL 14

1.5.2 Universal Coding and Refined MDL 17

1.5.3 Refined MDL for Model Selection 18

1.5.4 Refined MDL for Prediction and Hypothesis Selection 20

1.6 Some Remarks on Model Selection 23

1.6.1 Model Selection among Non-Nested Models 23

1.6.2 Goals of Model vs. Point Hypothesis Selection 25

1.7 The MDL Philosophy 26

1.8 MDL, Occam's Razor, and the "True Model"

29

1.8.1 Answer to Criticism No. 130 
1.8.2 Answer to Criticism No. $2 \quad 32$

1.9 History and Forms of MDL 36
1.9.1 What Is MDL? 37
1.9.2 MDL Literature 38

1.10 Summary and Outlook 40

2 Probabilistic and Statistical Preliminaries

41

2.1 General Mathematical Preliminaries 41

2.2 Probabilistic Preliminaries 46

2.2.1 Definitions; Notational Conventions 46

2.2.2 Probabilistic Sources 53

2.2.3 Limit Theorems and Statements 55

2.2.4 Probabilistic Models 57

2.2.5 Probabilistic Model Classes 60

2.3 Kinds of Probabilistic Models* 62

2.4 Terminological Preliminaries 69

2.5 Modeling Preliminaries:

Goals and Methods for Inductive Inference $\quad 71$

2.5.1 Consistency 71

2.5.2 Basic Concepts of Bayesian Statistics 74

2.6 Summary and Outlook 78

\section{Information-Theoretic Preliminaries 79}

3.1 Coding Preliminaries 79

3.1.1 Restriction to Prefix Coding Systems;

Descriptions as Messages 83

3.1.2 Different Kinds of Codes 86

3.1.3 Assessing the Efficiency of Description Methods

3.2 The Most Important Section of This Book:

Probabilities and Code Lengths 90

3.2.1 The Kraft Inequality 91

3.2.2 Code Lengths "Are" Probabilities 95

3.2.3 Immediate Insights and Consequences 99

3.3 Probabilities and Code Lengths, Part II 101

3.3.1 (Relative) Entropy and the Information Inequality

103

3.3.2 Uniform Codes, Maximum Entropy, and Minimax Codelength 106

3.4 Summary, Outlook, Further Reading 106 
4 Information-Theoretic Properties of Statistical Models

4.1 Introduction 109

4.2 Likelihood and Observed Fisher Information 111

4.3 KL Divergence and Expected Fisher Information 117

4.4 Maximum Likelihood: Data vs. Parameters 124

4.5 Summary and Outlook 130

5 Crude Two-Part Code MDL 131

5.1 Introduction: Making Two-Part MDL Precise 132

5.2 Two-Part Code MDL for Markov Chain Selection 133

5.2.1 The Code $C_{2} \quad 135$

5.2.2 The Code $C_{1} \quad 137$

5.2.3 Crude Two-Part Code MDL for Markov Chains 138

5.3 Simplistic Two-Part Code MDL Hypothesis Selection 139

5.4 Two-Part MDL for Tasks Other Than Hypothesis Selection 141

5.5 Behavior of Two-Part Code MDL 142

5.6 Two-Part Code MDL and Maximum Likelihood 144

5.6.1 The Maximum Likelihood Principle 144

5.6.2 MDL vs. ML 147

5.6.3 MDL as a Maximum Probability Principle 148

5.7 Computing and Approximating Two-Part MDL in Practice 150

5.8 Justifying Crude MDL: Consistency and Code Design 152

5.8.1 A General Consistency Result 153

5.8.2 Code Design for Two-Part Code MDL 157

5.9 Summary and Outlook 163

5.A Appendix: Proof of Theorem $5.1 \quad 163$

\section{Universal Coding 165}

6 Universal Coding with Countable Models 171

6.1 Universal Coding: The Basic Idea 172

6.1.1 Two-Part Codes as Simple Universal Codes 174

6.1.2 From Universal Codes to Universal Models 175

6.1.3 Formal Definition of Universality 177

6.2 The Finite Case 178

6.2.1 Minimax Regret and Normalized ML 179

6.2.2 NML vs. Two-Part vs. Bayes 182

6.3 The Countably Infinite Case 184 
6.3.1 The Two-Part and Bayesian Codes 184

6.3.2 The NML Code 187

6.4 Prequential Universal Models 190

6.4.1 Distributions as Prediction Strategies 190

6.4.2 Bayes Is Prequential; NML and Two-part Are Not

193

6.4.3 The Prequential Plug-In Model 197

6.5 Individual vs. Stochastic Universality* 199

6.5.1 Stochastic Redundancy 199

6.5.2 Uniformly Universal Models 201

6.6 Summary, Outlook and Further Reading 204

7 Parametric Models: Normalized Maximum Likelihood

7.1 Introduction 207

7.1.1 Preliminaries 208

7.2 Asymptotic Expansion of Parametric Complexity 211

7.3 The Meaning of $\int_{\Theta} \sqrt{\operatorname{det} I(\theta)} d \theta \quad 216$

7.3.1 Complexity and Functional Form 217

7.3.2 KL Divergence and Distinguishability 219

7.3.3 Complexity and Volume 222

7.3.4 Complexity and the Number of Distinguishable Distributions* 224

7.4 Explicit and Simplified Computations 226

8 Parametric Models: Bayes 231

8.1 The Bayesian Regret 231

8.1.1 Basic Interpretation of Theorem 8.1 233

8.2 Bayes Meets Minimax - Jeffreys' Prior 234

8.2.1 Jeffreys' Prior and the Boundary 237

8.3 How to Prove the Bayesian and NML Regret Theorems 239

8.3.1 Proof Sketch of Theorem 8.1 239

8.3.2 Beyond Exponential Families 241

8.3.3 Proof Sketch of Theorem 7.1 243

8.4 Stochastic Universality* 244

8.A Appendix: Proofs of Theorem 8.1 and Theorem $8.2 \quad 248$

9 Parametric Models: Prequential Plug-in 257

9.1 Prequential Plug-in for Exponential Families 257

9.2 The Plug-in vs. the Bayes Universal Model 262

9.3 More Precise Asymptotics 265 
9.4 Summary 269

10 Parametric Models: Two-Part 271

10.1 The Ordinary Two-Part Universal Model 271

10.1.1 Derivation of the Two-Part Code Regret 274

10.1.2 Proof Sketch of Theorem $10.1 \quad 277$

10.1.3 Discussion 282

10.2 The Conditional Two-Part Universal Code* 284

10.2.1 Conditional Two-Part Codes for Discrete

Exponential Families 286

10.2.2 Distinguishability and the Phase Transition* 290

10.3 Summary and Outlook 293

11 NML With Infinite Complexity 295

11.1 Introduction 295

11.1.1 Examples of Undefined NML Distribution 298

11.1.2 Examples of Undefined Jeffreys' Prior 299

11.2 Metauniversal Codes 301

11.2.1 Constrained Parametric Complexity 302

11.2.2 Meta-Two-Part Coding 303

11.2.3 Renormalized Maximum Likelihood* 306

11.3 NML with Luckiness 308

11.3.1 Asymptotic Expansion of LNML 312

11.4 Conditional Universal Models 316

11.4.1 Bayesian Approach with Jeffreys' Prior 317

11.4.2 Conditional NML 320

11.4.3 Liang and Barron's Approach 325

11.5 Summary and Remarks 329

11.A Appendix: Proof of Theorem 11.4329

12 Linear Regression 335

12.1 Introduction 336

12.1.1 Prelude: The Normal Location Family 338

12.2 Least-Squares Estimation 340

12.2.1 The Normal Equations 342

12.2.2 Composition of Experiments 345

12.2.3 Penalized Least-Squares 346

12.3 The Linear Model 348

12.3.1 Bayesian Linear Model $\mathcal{M}^{\mathbf{X}}$ with Gaussian Prior 354 
12.3.2 Bayesian Linear Models $\mathcal{M}^{\mathbf{X}}$ and $\mathcal{S}^{\mathbf{X}}$ with Noninformative Priors 359

12.4 Universal Models for Linear Regression 363

12.4.1 NML 363

12.4.2 Bayes and LNML 364

12.4.3 Bayes-Jeffreys and CNML 365

13 Beyond Parametrics 369

13.1 Introduction 370

13.2 CUP: Unions of Parametric Models 372

13.2.1 CUP vs. Parametric Models 375

13.3 Universal Codes Based on Histograms 376

13.3.1 Redundancy of Universal CUP Histogram Codes

13.4 Nonparametric Redundancy 383

13.4.1 Standard CUP Universal Codes 384

13.4.2 Minimax Nonparametric Redundancy 387

13.5 Gaussian Process Regression* 390

13.5.1 Kernelization of Bayesian Linear Regression 390

13.5.2 Gaussian Processes 394

13.5.3 Gaussian Processes as Universal Models 396

13.6 Conclusion and Further Reading 402

\section{Refined MDL 403}

14 MDL Model Selection 409

14.1 Introduction 409

14.2 Simple Refined MDL Model Selection 411

14.2.1 Compression Interpretation 415

14.2.2 Counting Interpretation 416

14.2.3 Bayesian Interpretation 418

14.2.4 Prequential Interpretation 419

14.3 General Parametric Model Selection 420

14.3.1 Models with Infinite Complexities 420

14.3.2 Comparing Many or Infinitely Many Models 422

14.3.3 The General Picture 425

14.4 Practical Issues in MDL Model Selection 428

14.4.1 Calculating Universal Codelengths 428 
14.4.2 Computational Efficiency and Practical Quality of Non-NML Universal Codes 429

14.4.3 Model Selection with Conditional NML and Plug-in Codes 431

14.4.4 General Warnings about Model Selection 435

14.5 MDL Model Selection for Linear Regression 438

14.5.1 Rissanen's RNML Approach 439

14.5.2 Hansen and Yu's gMDL Approach 443

14.5.3 Liang and Barron's Approach 446

14.5.4 Discussion 448

14.6 Worst Case vs. Average Case* 451

15 MDL Prediction and Estimation $\quad 459$

15.1 Introduction 459

15.2 MDL for Prediction and Predictive Estimation 460

15.2.1 Prequential MDL Estimators 461

15.2.2 Prequential MDL Estimators Are Consistent 465

15.2.3 Parametric and Nonparametric Examples 469

15.2.4 Césaro KL consistency vs. KL consistency* 472

15.3 Two-Part Code MDL for Point Hypothesis Selection 476

15.3.1 Discussion of Two-Part Consistency Theorem 478

15.4 MDL Parameter Estimation 483

15.4.1 MDL Estimators vs. Luckiness ML Estimators 487

15.4.2 What Estimator To Use? 491

15.4.3 Comparison to Bayesian Estimators* 493

15.5 Summary and Outlook 498

15.A Appendix: Proof of Theorem 15.3499

16 MDL Consistency and Convergence 501

16.1 Introduction 501

16.1.1 The Scenarios Considered 501

16.2 Consistency: Prequential and Two-Part MDL Estimators 502

16.3 Consistency: MDL Model Selection 505

16.3.1 Selection between a Union of Parametric Models 505

16.3.2 Nonparametric Model Selection Based on CUP Model Class 508

16.4 MDL Consistency Peculiarities 511

16.5 Risks and Rates 515 
16.5.1 Relations between Divergences and Risk Measures 517

16.5.2 Minimax Rates 519

16.6 MDL Rates of Convergence 520

16.6.1 Prequential and Two-Part MDL Estimators 520

16.6.2 MDL Model Selection 522

17 MDL in Context 523

17.1 MDL and Frequentist Paradigms 524

17.1.1 Sanity Check or Design Principle? 525

17.1.2 The Weak Prequential Principle 528

17.1.3 MDL vs. Frequentist Principles: Remaining Issues 529

17.2 MDL and Bayesian Inference 531

17.2.1 Luckiness Functions vs. Prior Distributions 534

17.2.2 MDL, Bayes, and Occam 539

17.2.3 MDL and Brands of Bayesian Statistics 544

17.2.4 Conclusion: a Common Future after All? 548

17.3 MDL, AIC and BIC 549

17.3.1 BIC 549

17.3.2 AIC 550

17.3.3 Combining the Best of AIC and BIC 552

17.4 MDL and MML 555

17.4.1 Strict Minimum Message Length 556

17.4.2 Comparison to MDL 558

17.4.3 The Wallace-Freeman Estimator 560

17.5 MDL and Prequential Analysis 562

17.6 MDL and Cross-Validation 565

17.7 MDL and Maximum Entropy 567

17.8 Kolmogorov Complexity and Structure Function 570

17.9 MDL and Individual Sequence Prediction 573

17.10 MDL and Statistical Learning Theory 579

17.10.1 Structural Risk Minimization 581

17.10.2 PAC-Bayesian Approaches 585

17.10.3 PAC-Bayes and MDL 588

17.11 The Road Ahead 592

\section{Additional Background 597}


18.1 Introduction 600

18.2 Definition and Overview 601

18.3 Basic Properties 605

18.4 Mean-Value, Canonical, and Other Parameterizations 609

18.4.1 The Mean Value Parameterization 609

18.4.2 Other Parameterizations 611

18.4.3 Relating Mean-Value and Canonical Parameters** 613

18.5 Exponential Families of General Probabilistic Sources* 617

18.6 Fisher Information Definitions and Characterizations* 619

19 Information-Theoretic Properties of Exponential Families 623

19.1 Introduction 624

19.2 Robustness of Exponential Family Codes 624

19.2.1 If $\Theta_{\text {mean }}$ Does Not Contain the Mean** 627

19.3 Behavior at the ML Estimate $\hat{\beta} \quad 629$

19.4 Behavior of the ML Estimate $\hat{\beta} \quad 632$

19.4.1 Central Limit Theorem 633

19.4.2 Large Deviations 634

19.5 Maximum Entropy and Minimax Codelength 637

19.5.1 Exponential Families and Maximum Entropy 638

19.5.2 Exponential Families and Minimax Codelength 641

19.5.3 The Compression Game 643

19.6 Likelihood Ratio Families and Rényi Divergences* 645

19.6.1 The Likelihood Ratio Family 647

19.7 Summary 650

References $\quad 651$

List of Symbols $\quad 675$

Subject Index 679 



\section{List of Figures}

1.1 A simple, a complex and a tradeoff (third-degree) polynomial. 13

1.2 Models and Model Classes; (Point) Hypotheses. 15

3.1 Coding systems, codes and description methods as defined in this book. MDL is only concerned with nonsingular codes/coding systems, allowing for lossless coding.

3.2 Binary code tree for the Kraft inequality using alphabet $\{a, b, c\}$ and code $C_{0}(a)=0 ; C_{0}(b)=10 ; C_{0}(c)=11$.

3.3 The most important observation of this book. 96

3.4 The third most important observation of this book. 107

4.1 The horizontal axis represents $\theta-\hat{\theta}\left(x^{n}\right)$ as a function of $\theta$ for a particular, fixed, $x^{n}$. The vertical axis represents $P_{\theta}\left(x^{n}\right)$. The function achieves its maximum at $\theta=\hat{\theta}$ and, near the maximum, has the shape of a Gaussian.

4.2 $I(\theta)$ as a function of $\theta$ for the Bernoulli model.

4.3 The horizontal axis represents $\theta$. The vertical axis represents $\mathrm{D}\left(\theta^{*} \| \theta\right)$ (solid thick line), $\mathrm{D}\left(\theta \| \theta^{*}\right)$ (solid thin line), and $0.5\left(\theta-\theta^{*}\right)^{2} I\left(\theta^{*}\right)$ (dotted line). In the upper picture, $\theta^{*}=0.5$. In the lower picture, $\theta^{*}=0.9$. 
4.4 The top graph shows the negative log-likelihood $-\nu \ln \theta-(1-\nu) \ln (1-\theta)$ as a function of $\theta$, where $\nu$ represents $n_{1} / n$. The graph shows the cases $\nu=0.5$ (line that is lowest on the left, highest on the right), $\nu=0.7$ (solid middle line), and $\nu=0.9$. Note that for $\nu=0.5, \theta$ achieves its minimum at $0.5: \hat{\theta}=\nu$. Similarly for $\nu=0.7, \hat{\theta}=0.7$, and for $\nu=0.9, \hat{\theta}=0.9$. Nevertheless, $\theta=0.7$ assigns a smaller description length to data with $\nu=0.9$ than to data with $\nu=0.7$. This is further illustrated in the bottom graph, which shows the negative log-likelihood $-\nu \ln \theta-(1-\nu) \ln (1-\theta)$ as a function of $\nu$, for $\theta=0.5, \theta=0.7$, and $\theta=0.9$. The corresponding functions are obviously linear. Note that we depict minus log rather than direct likelihoods here, which explains the difference in form between the top figure and the graph in Figure 4.1.

7.1 The crazy Bernoulli model.

10.1 The structure of the discretization for the case $\Theta \subset \mathbb{R}^{2}$. The picture shows a single "large" hypercube $S$ containing some "small" hyperrectangles $R$. The discretized points are the centers of the rectangles, if the rectangles lie completely inside $S$. Otherwise they are the closest points to the center that still lies within $S$. For the $S$ that is shown here, the angle between the small and the large grid is 30 degrees; for other "large" $S$, the angle of the $R \subset S$ will be different. The arrows point in the direction of the eigenvectors of the Fisher information matrix. The length of the arrows is proportional to the square root of the inverse of the eigenvalues.

14.1 The Refined MDL Principle for Model Selection 426

14.2 Ignoring codelengths.

17.1 Rissanen's MDL, Wallace's MML and Dawid's Prequential Approach 


\section{Series Foreword}

The goal of building systems that can adapt to their environments and learn from their experience has attracted researchers from many fields, including computer science, engineering, mathematics, physics, neuroscience, and cognitive science. Out of this research has come a wide variety of learning techniques that have the potential to transform many scientific and industrial fields. Recently, several research communities have converged on a common set of issues surrounding supervised, unsupervised and reinforcement learning problems. The MIT Press series on Adaptive Computation and Machine Learning seeks to unify the many diverse strands of machine learning research and to foster high-quality research and innovative applications.

Thomas Dietterich 



\section{Foreword}

This is a splendid account of the latest developments on the minimum description length (MDL) principle and the related theory of stochastic complexity. The MDL principle seeks to place the age-old statistical or inductive inference on a sound foundation. In order to achieve this it requires the drastically different and, for many, unpalatable view that the objective is not to estimate any "true" data-generating mechanism but simply to find a good explanation of data, technically called a model. The author gives an impassionate balanced discussion of the deep philosophical implications of the principle, and he traces the tortuous path from the roots to the current refined stage of the principle, in which the idea of a universal model plays a central role. This is a model that allows for an objective comparison of alternative models regardless of their form or number of parameters in case the interest is in model selection. Further, it provides a basis for prediction and classification.

The author describes painstakingly the information- and probability-theoretic notions needed for the reader with a minimum of prerequisites to apply the principle to a variety of statistical problems. This involves an in-depth treatment of the theory of "universal models," which in its general form is deep and complex. The author's treatment of it, however, is highly accessible. He achieves this by devoting an extensive section on discussing finite universal models, which are much simpler than the general case but do serve to illustrate the general ideas.

Based on this treatment, he then introduces the MDL principle in its modern, refined form, always emphasizing the ideas that give rise to the actual formulas. He starts out with the simple case of comparing a finite number of parametric models, and gradually builds up the theory to general problems of model selection. He also briefly discusses parameter estimation and 
nonparametric inference. For the reader with deeper statistical knowledge, in Chapter 17 he compares MDL to some other more customary statistical techniques.

\author{
Jorma Rissanen \\ Helsinki Institute for Information Technology \\ Helsinki, Finland \\ December 2005
}




\section{Preface}

How does one decide among competing explanations of data given limited observations? This is the problem of model selection. A central concern in model selection is the danger of overfitting: the selection of an overly complex model that, while fitting observed data very well, predicts future data very badly. Overfitting is one of the most important issues in inductive and statistical inference: besides model selection, it also pervades applications such as prediction, pattern classification and parameter estimation.

The minimum description length (MDL) principle is a relatively recent method for inductive inference that provides a generic solution to the model selection problem, and, more generally, to the overfitting problem. MDL is based on the following insight: any regularity in the data can be used to compress the data, i.e. to describe it using fewer symbols than the number of symbols needed to describe the data literally. The more regularities there are, the more the data can be compressed. Equating "learning" with "finding regularity," we can therefore say that the more we are able to compress the data, the more we have learned about the data. Formalizing this idea leads to a general theory of inductive inference with several attractive properties:

1. Occam's razor. MDL chooses a model that trades off goodness-of-fit on the observed data with "complexity" or "richness" of the model. As such, MDL embodies a form of Occam's razor, a principle that is both intuitively appealing and informally applied throughout all the sciences.

2. No overfitting, automatically. MDL methods automatically and inherently protect against overfitting and can be used to estimate both the parameters and the structure (e.g., number of parameters) of a model. In contrast, to avoid overfitting when estimating the structure of a model, traditional 
methods such as maximum likelihood must be modified and extended with additional, typically ad hoc principles.

3. Bayesian interpretation. Some (not all) MDL procedures are closely related to Bayesian inference. Yet they avoid some of the interpretation difficulties of the Bayesian approach, especially in the realistic case when it is known a priori to the modeler that none of the models under consideration is true. In fact:

4. No need for "underlying truth." In contrast to other statistical methods, MDL procedures have a clear interpretation independent of whether or not there exists some underlying "true" model.

5. Predictive interpretation. Because data compression is formally equivalent to a form of probabilistic prediction, MDL methods can be interpreted as searching for a model with good predictive performance on unseen data. This makes MDL related to, yet different from, data-oriented model selection techniques such as cross-validation.

\section{This Book}

This book provides an extensive, step-by-step introduction to the MDL principle, with an emphasis on conceptual issues. From the many talks that I have given on the subject, I have noticed that the same questions about MDL pop up over and over again. Often, the corresponding answers can be found only - if at all - in highly technical journal articles. The main aim of this book is to serve as a reference guide, in which such answers can be found in a much more accessible form. There seems to be a real need for such an exposition because, quoting Lanterman (2001), of "the challenging nature of the original works and the preponderance of misinterpretations and misunderstandings in the applied literature." Correcting such misunderstandings is the second main aim of this book.

First Aim: Accessibility I first learned about MDL in 1993, just before finishing my master's in computer science. As such, I knew some basic probability theory and linear algebra, but I knew next to nothing about advanced measure-theoretic probability, statistics, and information theory. To my surprise, I found that to access the MDL literature, I needed substantial knowledge about all three subjects! This experience has had a profound influence on this book: in a way, I wanted to write a book which I would have been 
able to understand when I was a beginning graduate student. Therefore, since with some difficulty its use can be avoided, there is no measure theory whatsoever in this book. On the other hand, this book is full of statistics and information theory, since these are essential to any understanding of MDL. Still, both subjects are introduced at a very basic level in Part I of the book, which provides an initial introduction to MDL. At least this part of the book should be readable without any prior exposure to statistics or information theory.

If my main aim has succeeded, then this book should be accessible to (a) researchers from the diverse areas dealing with inductive inference, such as statistics, pattern classification, and branches of computer science such as machine learning and data mining; (b) researchers from biology, econometrics, experimental psychology, and other applied sciences that frequently have to deal with inductive inference, especially model selection; and (c) philosophers interested in the foundations of inductive inference. This book should enable such readers to understand what MDL is, how it can be used, and what it does.

Second Aim: A Coherent, Detailed Overview In the year 2000, when I first thought about writing this book, the field had just witnessed a number of advances and breakthroughs, involving the so-called normalized maximum likelihood code. These advances had not received much attention outside of a very small research community; most practical applications and assessments of MDL were based on "old" (early 1980s) methods and ideas. At the time, some pervasive myths were that "MDL is just two-part coding", "MDL is BIC" (an asymptotic Bayesian method for model selection), or "MDL is just Bayes." This prompted me and several other researchers to write papers and give talks about the new ideas, related to the normalized maximum likelihood. Unfortunately, this may have had somewhat of an adverse effect: I now frequently talk to people who think that MDL is just "normalized maximum likelihood coding." This is just as much of a myth as the earlier ones! In reality, MDL in its modern form is based on a general notion known in the information-theoretic literature as universal coding. There exist many types of universal codes, the main four types being the Bayesian, two-part, normalized maximum likelihood, and prequential plug-in codes. All of these can be used in MDL inference, and which one to use depends on the application at hand. While this emphasis on universal codes is already present in the overview (Barron, Rissanen, and Yu 1998), their paper requires substan- 
tial knowledge of information theory and statistics. With this book, I hope to make the universal coding-based MDL theory accessible to a much wider audience.

\section{A Guide for the Reader}

This book consists of four parts. Part I is really almost a separate book. It provides a very basic introduction to MDL, as well as an introductory overview of the statistical and information-theoretic concepts needed to understand MDL. Part II is entirely devoted to universal coding, the information-theoretic notion on which MDL is built. Universal coding is really a theory about data compression. It is easiest to introduce without directly connecting it to inductive inference, and this is the way we treat it in Part II. In fact though, there is a very strong relation between universal coding and inductive inference. This connection is formalized in Part III, where we give a detailed treatment of MDL theory as a theory of inductive inference based on universal coding. Part IV can once again be read separately, providing an overview of the statistical theory of exponential families. It provides background knowledge needed in the proofs of theorems in Part II.

The Fast Track - How to Avoid Reading Most of This Book I do not suppose that any reader will find the time to read all four parts in detail. Indeed, for readers with prior exposure to MDL, this book may serve more like a reference guide than an introduction in itself. For the benefit of readers with no such prior knowledge, each chapter in Part I and Part II starts with a brief list of its contents as well as a fast track-paragraph, which indicates the parts that should definitely be read, and the parts that can be skipped at first reading. This allows a "fast track" through Part I and Part II, so that the reader can quickly reach Part III, which treats state-of-the-art MDL inference. Additionally, some sections are marked with an asterisk $\left({ }^{*}\right)$. Such sections contain advanced material and may certainly be skipped at first reading.

Also, the reader will frequently find paragraphs such as the present one, which are set in smaller font. These provide additional, more detailed discussion of the issues arising in the main text, and may also be skipped at first reading.

Also, at several places, the reader will find boxes like the one below: 


\section{Boxes Contain the Most Important Ideas}

Each chapter contains several boxes like this one. These contain the most important insights. Together, they form a summary of the chapter.

To further benefit the hurried reader, we now give a brief overview of each part:

Part I Chapter 1 discusses some of the basic ideas underlying MDL in a mostly nonmathematical manner. Chapter 2 briefly reviews general mathematical and probabilistic preliminaries. Chapter 3 gives a detailed discussion of some essential information-theoretic ideas. Chapter 4 applies these notions to statistical models. This chapter gives an extensive analysis of the log-likelihood function and its expectation. It may be of interest for teachers of introductory statistics, since the treatment emphasizes some, in my view, quite important aspects usually not considered in statistics textbooks. For example, we consider in detail what happens if we vary the data, rather than the parameters. Chapter 5 then gives a first mathematically precise implementation of MDL. This is the so-called crude two-part code MDL. I call it "crude" because it is suboptimal, and not explicitly based on universal coding. I included it because it is easy to explain - especially the fact that it has obvious defects raises some serious questions, and thinking about these questions seems the perfect introduction to the "refined" MDL that we introduce in Part III of the book.

Although some basic familiarity with elementary probability theory is assumed throughout the text, all probabilistic concepts needed are briefly reviewed in Chapter 2. They are typically taught in undergraduate courses and can be found in books such as (Ross 1998). Strictly speaking, the text can be read without any prior knowledge of statistics or information theory - all concepts and ideas are introduced in Chapters 3 and 4 . Nevertheless, some prior exposure to these subjects is probably needed to fully appreciate the developments in Part II and Part III. More extensive introductions to the statistical concepts needed can be found in, for example (Bain and Engelhardt 1989; Casella and Berger ; Rice 1995).

Part II Part II then treats the general theory of universal coding, with an emphasis on issues that are relevant to MDL. It starts with a brief introduction which gives a high-level overview of the chapters contained in Part II. Its first chapter, Chapter 6, then contains a detailed introduction to the main 
ideas, in the restricted context of countable model classes. Each of the four subsequent chapters gives a detailed discussion of one of the four main types of universal codes, in the still restricted context of "parametric models" with (essentially) compact parameter spaces. Chapters 11,12, and 13 deal with general parametric models - including linear regression models - as well as nonparametric models.

Part III Part III gives a detailed treatment of refined MDL. We call it "refined" so as to mark the contrast with the "crude" form of MDL of Chapter 5. It starts with a brief introduction which gives a high-level overview of refined MDL. Chapter 14 deals with refined MDL for model selection. Chapter 15 is about its other two main applications: hypothesis selection (a basis for parametric and nonparametric density estimation) and prediction. Consistency and rate-of-convergence results for refined MDL are detailed in Chapter 16. Refined MDL is placed in its proper context in Chapter 17, in which we discuss its underlying philosophy and compare it to various other approaches.

Compared to Part I, Part II and Part III contain more advanced material, and some prior exposure to statistics may be needed to fully appreciate the developments. Still, all required information-theoretic concepts - invariably related to universal coding - are once again discussed at a very basic level. These parts of the book mainly serve as a reference guide, providing a detailed exposition of the main topics in MDL inference. The discussion of each topic includes details which are often left open in the existing literature, but which are important when devising practical applications of MDL. When pondering these details, I noticed that there are several open questions in MDL theory which previously have not been explicitly posed. We explicitly list and number such open questions in Part II and Part III. These parts also contain several new developments: in order to tell a coherent story about MDL, I provide some new results - not published elsewhere - that connect various notions devised by different authors.

The main innovations are the "distinguishability" interpretation of MDL for finite models in Chapter 6, the "phase transition" view on two-part coding in Chapter 10, the luckiness framework as well as the CNML-1 and CNML2 extensions of the normalized maximum likelihood code in Chapter 11, and the connections between Césaro and standard KL risk and the use of redundancy rather than resolvability in the convergence theorem for two-part MDL in Chapter 15. 
I also found it useful to rephrase and re-prove existing mathematical theorems in a unified way. The many theorems in Part II and Part III usually express results that are similar to existing theorems by various authors, mainly Andrew Barron, Jorma Rissanen, and Bin Yu. Since these theorems were often stated in slightly different contexts, they are hard to compare. In our version, they become easily comparable. Specifically, in Part II, we restrict the treatment to so-called exponential families of distributions, which is a weakening of existing results. Yet, the theorems invariably deal with uniform convergence, which is often a strengthening of existing results.

Part IV: Exponential Family Theory The theorems in Part II make heavy use of the general and beautiful theory of exponential or, relatedly, maximum entropy families of probability distributions. Part IV is an appendix that contains an overview of these families and their mathematical properties. When writing the book, I found that most existing treatments are much too restricted to contain the results that we need in this book. The only general treatments I am aware of (Barndorff-Nielsen 1978; Brown 1986) use measure theory, and give a detailed treatment of behavior at parameters tending to the boundaries of the parameter space. For this reason, they are quite hard to follow. Thus, I decided to write my own overview, which avoids measure theory and boundary issues, but otherwise contains most essential ideas such as sufficiency, mean-value and canonical parameterizations, duality, and maximum entropy interpretations.

\section{Acknowledgments}

Tim van Erven, Peter Harremoës, Wouter Koolen, In Jae Myung, Mark Pitt, Teemu Roos, Steven de Rooij, and Tomi Silander read and commented on parts of this text. I would especially like to thank Tim, who provided comments on the entire manuscript.

\section{Mistakes}

Of course, the many mistakes which undoubtedly remain in this text are all my (the author's) sole responsibility. I welcome all emails that point out mistakes in the text!

Among those who have helped shape my views on statistical inference, two people stand out: Phil Dawid and Jorma Rissanen. Other people who have 
strongly influenced my thinking on these matters are Vijay Balasubramanian, Andrew Barron, Richard Gill, Teemu Roos, Paul Vitányi, Volodya Vovk, and Eric-Jan Wagenmakers. My wife Louise de Rooij made a very visible and colourful contribution. Among the many other people who in some way or other had an impact on this book I should mention Petri Myllymäki, Henry Tirri, Richard Shiffrin, Johan van Benthem, and, last but not least, Herbert, Christa and Wiske Grünwald. As leaders of our research group at CWI (the National Research Institute for Mathematics and Computer Science in the Netherlands), Harry Buhrman and Paul Vitányi provided the pleasant working environment in which this book could be written. The initial parts of this book were written in 2001, while I was visiting the University of California at Santa Cruz. I would like to thank Manfred Warmuth and David Draper for hosting me. Finally and most importantly, I would like to thank my lovely wife Louise for putting up with my foolishness for so long. 


\section{PART I}

\section{Introductory Material}





\section{Learning, Regularity, and Compression}

Overview The task of inductive inference is to find laws or regularities underlying some given set of data. These laws are then used to gain insight into the data or to classify or predict future data. The minimum description length (MDL) principle is a general method for inductive inference, based on the idea that the more we are able to compress (describe in a compact manner) a set of data, the more regularities we have found in it and therefore, the more we have learned from the data. In this chapter we give a first, preliminary and informal introduction to this principle.

Contents In Sections 1.1 and 1.2 we discuss some of the fundamental ideas relating description length and regularity. In Section 1.3 we describe what was historically the first attempt to formalize these ideas. In Section 1.4 we explain the problems with using the original formalization in practice, and indicate what must be done to make the ideas practicable. Section 1.5 introduces the practical forms of MDL we deal with in this book, as well as the crucial concept of "universal coding." Section 1.6 deals with some issues concerning model selection, which is one of the main MDL applications. The philosophy underlying MDL is discussed in Section 1.7. Section 1.8 shows how the ideas behind MDL are related to "Occam's razor." We end in Section 1.9 with a brief historical overview of the field and its literature.

Fast Track This chapter discusses, in an informal manner, several of the complicated issues we will deal with in this book. It is therefore essential for readers without prior exposure to MDL. Readers who are familiar with the basic ideas behind MDL may just want to look at the boxes. 


\subsection{Regularity and Learning}

We are interested in developing a method for learning the laws and regularities in data. The following example will illustrate what we mean by this and give a first idea of how it can be related to descriptions of data.

Example 1.1 We start by considering binary data. Consider the following three sequences. We assume that each sequence is 10000 bits long, and we just list the beginning and the end of each sequence.

$$
\begin{aligned}
& 00010001000100010001 \ldots 0001000100010001000100010001 \\
& 01110100110100100110 \ldots 1010111010111011000101100010 \\
& 00011000001010100000 \ldots 0010001000010000001000110000
\end{aligned}
$$

The first of these three sequences is a 2500-fold repetition of 0001 . Intuitively, the sequence looks regular; there seems to be a simple "law" underlying it; it might make sense to conjecture that future data will also be subject to this law, and to predict that future data will behave according to this law. The second sequence has been generated by tosses of a fair coin. It is, intuitively speaking, as "random as possible," and in this sense there is no regularity underlying it. ${ }^{1}$ Indeed, we cannot seem to find such a regularity either when we look at the data. The third sequence contains exactly four times as many 0 s as 1 s. It looks less regular, more random than the first; but it looks less random than the second. There is still some discernible regularity in these data, but of a statistical rather than of a deterministic kind. Again, noticing that such a regularity is there and predicting that future data will behave according to the same regularity seems sensible.

\subsection{Regularity and Compression}

What do we mean by a "regularity"? The fundamental idea behind the MDL principle is the following insight: every regularity in the data can be used to compress the data, i.e. to describe it using fewer symbols than the number of symbols needed to describe the data literally. Such a description should always uniquely specify the data it describes - hence given a description or

1. Unless we call "generated by a fair coin toss" a "regularity" too. There is nothing wrong with that view - the point is that, the more we can compress a sequence, the more regularity we have found. One can avoid all terminological confusion about the concept of "regularity" by making it relative to something called a "base measure," but that is beyond the scope of this book (Li and Vitányi 1997). 
encoding $D^{\prime}$ of a particular sequence of data $D$, we should always be able to fully reconstruct $D$ using $D^{\prime}$.

For example, sequence (1.1) above can be described using only a few words; we have actually done so already: we have not given the complete sequence - which would have taken about the whole page - but rather just a onesentence description of it that nevertheless allows you to reproduce the complete sequence if necessary. Of course, the description was done using natural language and we may want to do it in some more formal manner.

If we want to identify regularity with compressibility, then it should also be the case that nonregular sequences can not be compressed. Since sequence (1.2) has been generated by fair coin tosses, it should not be compressible. As we will show below, we can indeed prove that whatever description method $C$ one uses, the length of the description of a sequence like (1.2) will, with overwhelming probability, be not much shorter than sequence (1.2) itself.

Note that the description of sequence (1.3) that we gave above does not uniquely define sequence (1.3). Therefore, it does not count as a "real" description: one cannot regenerate the whole sequence if one has the description. A unique description that still takes only a few words may look like this: "Sequence (1.3) is one of those sequences of 10000 bits in which there are four times as many $0 \mathrm{~s}$ as there are 1s. In the lexicographical ordering of those sequences, it is number $i$." Here $i$ is some large number that is explicitly spelled out in the description. In general, there are $2^{n}$ binary sequences of length $n$, while there are only $\left(\begin{array}{c}n \\ \nu n\end{array}\right)$ sequences of length $n$ with a fraction of $\nu$ 1s. For every rational number $\nu$ except $\nu=1 / 2$, the ratio of $\left(\begin{array}{c}n \\ \nu n\end{array}\right)$ to $2^{n}$ goes to 0 exponentially fast as $n$ increases (this is shown formally in Chapter 4; see Equation (4.36) on page 129 and the text thereunder; by the method used there one can also show that for $\nu=1 / 2$, it goes to 0 as $O(1 / \sqrt{n})$ ). It follows that compared to the total number of binary sequences of length 10000, the number of sequences of length 10000 with four times as many 0s as 1 s is vanishingly small. Direct computation shows it is smaller than $2^{7213}$, so that the ratio between the number of sequences with four times as many 0 s than 1 s and the total number of sequences is smaller than $2^{-2787}$. Thus, $i<2^{7213} \ll 2^{10000}$ and to write down $i$ in binary we need approximately $\left(\log _{2} i\right)<7213 \ll 10000$ bits.

Example 1.2 [Compressing Various Regular Sequences] The regularities underlying sequences (1) and (3) were of a very particular kind. To illustrate that any type of regularity in a sequence may be exploited to compress that sequence, we give a few more examples: 
The Number $\pi$ Evidently, there exists a computer program for generating the first $n$ digits of $\pi$ - such a program could be based, for example, on an infinite series expansion of $\pi$. This computer program has constant size, except for the specification of $n$ which takes no more than $O(\log n)$ bits. Thus, when $n$ is very large, the size of the program generating the first $n$ digits of $\pi$ will be very small compared to $n$ : the $\pi$-digit sequence is deterministic, and therefore extremely regular.

Physics Data Consider a two-column table where the first column contains numbers representing various heights from which an object was dropped. The second column contains the corresponding times it took for the object to reach the ground. Assume both heights and times are recorded to some finite precision. In Section 1.5 we illustrate that such a table can be substantially compressed by first describing the coefficients of the second-degree polynomial $H$ that expresses Newton's law; then describing the heights; and then describing the deviation of the time points from the numbers predicted by $H$.

Natural Language Most sequences of words are not valid sentences according to the English language. This fact can be exploited to substantially compress English text, as long as it is syntactically mostly correct: by first describing a grammar for English, and then describing an English text $D$ with the help of that grammar (Grünwald 1996), $D$ can be described using much less bits than are needed without the assumption that word order is constrained.

Description Methods In order to formalize our idea, we have to replace the part of the descriptions above that made use of natural language by some formal language. For this, we need to fix a description method that maps sequences of data to their descriptions. Each such sequence will be encoded as another sequence of symbols coming from some finite or countably infinite coding alphabet. An alphabet is simply a countable set of distinct symbols. An example of an alphabet is the binary alphabet $\mathbb{B}=\{0,1\}$; the three data sequences above are sequences over the binary alphabet. A sequence over a binary alphabet will also be called a binary string. Sometimes our data will consist of real numbers rather than binary strings. In practice, however, such numbers are always truncated to some finite precision. We can then again model them as symbols coming from a finite data alphabet.

More precisely, we are given a sample or equivalently data sequence $D=$ $\left(x_{1}, \ldots, x_{n}\right)$ where each $x_{i}$ is a member of some set $\mathcal{X}$, called the space of observations or the sample space for one observation. The set of all potential samples of length $n$ is denoted $\mathcal{X}^{n}$ and is called the sample space. We call 
$x_{i}$ a single observation or, equivalently, a data item. For a general note about how our terminology relates to the usual terminology in statistics, machine learning and pattern recognition, we refer to the box on page 72 .

Without any loss of generality we may describe our data sequences as binary strings (this is explained in Chapter 3, Section 3.2.2). Hence all the description methods we consider map data sequences to sequences of bits. All description methods considered in MDL satisfy the unique decodability property: given a description $D^{\prime}$, there is at most one ("unique") $D$ that is encoded as $D^{\prime}$. Therefore, given any description $D^{\prime}$, one should be able to fully reconstruct the original sequence $D$. Semiformally:

\section{Description Methods}

Definition 1.1 A description method is a one-many relation from the sample space to the set of binary strings of arbitrary length.

A truly formal definition will be given in Chapter 3, Section 3.1. There we also explain how our notion of "description method" relates to the more common and closely related notion of a "code." Until then, the distinction between codes an description methods is not that important, and we use the symbol $C$ to denote both concepts.

Compression and Small Subsets We are now in a position to show that strings which are "intuitively" random cannot be substantially compressed. We equate intuitively random with "having been generated by independent tosses of a fair coin." We therefore have to prove that it is virtually impossible to substantially compress sequences that have been generated by fair coin tosses. By "it is virtually impossible" we mean "it happens with vanishing probability." Let us take some arbitrary but fixed description method $C$ over the data alphabet consisting of the set of all binary sequences of length $\geq 1$. Such a code maps binary strings to binary strings. Suppose we are given a data sequence of length $n$ (in Example 1.1, $n=10000$ ). Clearly, there are $2^{n}$ possible data sequences of length $n$. We see that only two of these can be mapped to a description of length 1 (since there are only two binary strings of length 1: 0 and 1). Similarly, only a subset of at most $2^{m}$ sequences can have a description of length $m$. This means that at most $\sum_{i=1}^{m} 2^{i}<2^{m+1}$ data sequences can have a description length $\leq m$. The fraction of data sequences of length $n$ that can be compressed by more than $k$ bits is therefore at 
most $2^{-k}$ and as such decreases exponentially in $k$. If data are generated by $n$ tosses of a fair coin, then all $2^{n}$ possibilities for the data are equally probable, so the probability that we can compress the data by more than $k$ bits is smaller than $2^{-k}$. For example, the probability that we can compress the data by more than 20 bits is smaller than one in a million.

\section{Most Data Sets Are Incompressible}

Suppose our goal is to encode a binary sequence of length $n$. Then

- No matter what description method we use, only a fraction of at most $2^{-k}$ sequences can be compressed by more than $k$ bits.

- Thus, if data are generated by fair coin tosses, then no matter what code we use, the probability that we can compress a sequence by more than $k$ bits is at most $2^{-k}$.

- This observation will be generalized to data generated by an arbitrary distribution in Chapter 3. We then call it the no-hypercompression inequality. It can be found in the box on page 103 .

Seen in this light, having a short description length for the data is equivalent to identifying the data as belonging to a tiny, very special subset out of all a priori possible data sequences; see also the box on page 31 .

\subsection{Solomonoff's Breakthrough - Kolmogorov Complexity}

It seems that what data are compressible and what are not is extremely dependent on the specific description method used. In 1964 - in a pioneering paper that may be regarded as the starting point of all MDL-related research (Solomonoff 1964) - Ray Solomonoff suggested the use of a universal computer language as a description method. By a universal language we mean a computer language in which a universal Turing machine can be implemented. All commonly used computer languages, like Pascal, LISP, C, are "universal." Every data sequence $D$ can be encoded by a computer program $P$ that prints $D$ and then halts. We can define a description method that maps each data sequence $D$ to the shortest program that prints $D$ and then 
halts. ${ }^{2}$ Clearly, this is a description method in our sense of the word in that it defines a 1-many (even 1-1) mapping from sequences over the data alphabet to a subset of the binary sequences.

The shortest program for a sequence $D$ is then interpreted as the optimal hypothesis for $D$. Let us see how this works for sequence (1.1) above. Using a language similar to $C$, we can write a program

$$
\text { for } i=1 \text { to } 2500 \text {; do }\left\{\text { print }{ }^{\prime} 0001^{\prime}\right\} \text {; halt }
$$

which prints sequence (1.1) but is clearly a lot shorter than it. If we want to make a fair comparison, we should rewrite this program in a binary alphabet; the resulting number of bits is still much smaller than 10000. The shortest program printing sequence (1.1) is at least as short as the program above, which means that sequence (1.1) is indeed highly compressible using Solomonoff's code. By the arguments of the previous section we see that, given an arbitrary description method $C$, sequences like (1.2) that have been generated by tosses of a fair coin are very likely not substantially compressible using $C$. In other words, the shortest program for sequence (1.1) is, with extremely high probability, not much shorter than the following:

$$
\text { print '01110100110100001010.......10111011000101100010'; halt }
$$

This program has size about equal to the length of the sequence. Clearly, it is nothing more than a repetition of the sequence.

Kolmogorov Complexity We define the Kolmogorov complexity of a sequence as the length of the shortest program that prints the sequence and then halts. Kolmogorov complexity has become a large subject in its own right; see ( $\mathrm{Li}$ and Vitányi 1997) for a comprehensive introduction.

The lower the Kolmogorov complexity of a sequence, the more regular or equivalently, the less random, or, yet equivalently, the simpler it is. Measuring regularity in this way confronts us with a problem, since it depends on the particular programming language used. However, in his 1964 paper, Ray Solomonoff (Solomonoff 1964) showed that asymptotically it does not matter what programming language one uses, as long as it is universal: for every sequence of data $D=\left(x_{1}, \ldots, x_{n}\right)$, let us denote by $L_{\mathrm{UL}}(D)$ the length of the shortest program for $D$ using universal language UL. We can show that for

2. If there exists more than one shortest program, we pick the one that comes first in enumeration order. 
every two universal languages $\mathrm{UL}_{1}$ and $\mathrm{UL}_{2}$, the difference between the two lengths $L_{\mathrm{UL}_{1}}(D)-L_{\mathrm{UL}_{2}}(D)$ is bounded by a constant that depends on $\mathrm{UL}_{1}$ and $\mathrm{UL}_{2}$ but not on the length $n$ of the data sequence $D$. This implies that if we have a lot of data ( $n$ is large), then the difference in the two description lengths is negligible compared to the size of the data sequence. This result is known as the invariance theorem and was proved independently in (Solomonoff 1964), (Kolmogorov 1965) (hence the name Kolmogorov complexity), and (Chaitin 1969). The proof is based on the fact that one can write a compiler for every universal language $\mathrm{UL}_{1}$ in every other universal language $\mathrm{UL}_{2}$. Such a compiler is a computer program with length $L_{1 \rightarrow 2}$. For example, we can write a program in Pascal that translates every $C$ program into an equivalent Pascal program. The length (in bits) of this program would then be $L_{\mathrm{C} \rightarrow \text { Pascal }}$. We can simulate each program $P_{1}$ written in language $\mathrm{UL}_{1}$ by program $P_{2}$ written in $\mathrm{UL}_{2}$ as follows: $P_{2}$ consists of the compiler from $\mathrm{UL}_{1}$ to $\mathrm{UL}_{2}$, followed by $P_{1}$. The length of program $P_{2}$ is bounded by the length of $P_{1}$ plus $L_{1 \rightarrow 2}$. Hence for all data $D$, the maximal difference between $L_{\mathrm{UL}_{1}}(D)$ and $L_{\mathrm{UL}_{2}}(D)$ is bounded by $\max \left\{L_{1 \rightarrow 2}, L_{2 \rightarrow 1}\right\}$, a constant which only depends on $\mathrm{UL}_{1}$ and $\mathrm{UL}_{2}$ but not on $D$.

\subsection{Making the Idea Applicable}

Problems There are two major problems with applying Kolmogorov complexity to practical learning problems:

1. Uncomputability. The Kolmogorov complexity cannot be computed in general;

2. Large constants. The description length of any sequence of data involves a constant depending on the description method used.

By "Kolmogorov complexity cannot be computed" we mean the following: there is no computer program that, for every sequence of data $D$, when given $D$ as input, returns the shortest program that prints $D$ and halts. Neither can there be a program, that for every data $D$ returns only the length of the shortest program that prints $D$ and then halts. Assuming such a program exists leads to a contradiction (Li and Vitányi 1997).

The second problem relates to the fact that in many realistic settings, we are confronted with very small data sequences for which the invariance theorem is not very relevant since the length of $D$ is small compared to the constant $L_{1 \rightarrow 2}$. 
"Idealized" or "Algorithmic" MDL If we ignore these problems, we may use Kolmogorov complexity as our fundamental concept and build a theory of idealized inductive inference on top of it. This road has been taken by Solomonoff $(1964,1978)$, starting with the 1964 paper in which he introduced Kolmogorov complexity, and by Kolmogorov, when he introduced the Kolmogorov minimum sufficient statistic (Li and Vitányi 1997; Cover and Thomas 1991). Both Solomonoff's and Kolmogorov's ideas have been substantially refined by several authors. We mention here P. Vitányi (Li and Vitányi 1997; Gács, Tromp, and Vitányi 2001; Vereshchagin and Vitányi 2002; Vereshchagin and Vitányi 2004; Vitányi 2005), who concentrated on Kolmogorov's ideas, and M. Hutter (2004), who concentrated on Solomonoff's ideas. Different authors have used different names for this area of research: "ideal MDL," "idealized MDL," or "algorithmic statistics." It is closely related to the celebrated theory of random sequences due to P. Martin-Löf and Kolmogorov (Li and Vitányi 1997). We briefly return to idealized MDL in Chapter 17, Section 17.8 .

Practical MDL Like most authors in the field, we concentrate here on nonidealized, practical versions of MDL that explicitly deal with the two problems mentioned above. The basic idea is to scale down Solomonoff's approach so that it does become applicable. This is achieved by using description methods that are less expressive than general-purpose computer languages. Such description methods $C$ should be restrictive enough so that for any data sequence $D$, we can always compute the length of the shortest description of $D$ that is attainable using method $C$; but they should be general enough to allow us to compress many of the intuitively "regular" sequences. The price we pay is that, using the "practical" MDL principle, there will always be some regular sequences which we will not be able to compress. But we already know that there can be no method for inductive inference at all which will always give us all the regularity there is - simply because there can be no automated method which for any sequence $D$ finds the shortest computer program that prints $D$ and then halts. Moreover, it will often be possible to guide a suitable choice of $C$ by a priori knowledge we have about our problem domain. For example, below we consider a description method $C$ that is based on the class of all polynomials, such that with the help of $C$ we can compress all data sets which can meaningfully be seen as points on some polynomial. 


\subsection{Crude MDL, Refined MDL and Universal Coding}

Let us recapitulate our main insights so far:

\section{MDL: The Basic Idea}

The goal of statistical inference may be cast as trying to find regularity in the data. "Regularity" may be identified with "ability to compress." MDL combines these two insights by viewing learning as data compression: it tells us that, for a given set of hypotheses $\mathcal{H}$ and data set $D$, we should try to find the hypothesis or combination of hypotheses in $\mathcal{H}$ that compresses $D$ most.

This idea can be applied to all sorts of inductive inference problems, but it turns out to be most fruitful in (and its development has mostly concentrated on) problems of model selection and, more generally, dealing with overfitting. Here is a standard example (we explain the difference between "model" and "hypothesis" after the example).

Example 1.3 [Model Selection and Overfitting] Consider the points in Figure 1.1. We would like to learn how the $y$-values depend on the $x$-values. To this end, we may want to fit a polynomial to the points. Straightforward linear regression will give us the leftmost polynomial - a straight line that seems overly simple: it does not capture the regularities in the data well. Since for any set of $n$ points there exists a polynomial of the $(n-1)$ st degree that goes exactly through all these points, simply looking for the polynomial with the least error will give us a polynomial like the one in the second picture. This polynomial seems overly complex: it reflects the random fluctuations in the data rather than the general pattern underlying it. Instead of picking the overly simple or the overly complex polynomial, it seems more reasonable to prefer a relatively simple polynomial with small but nonzero error, as in the rightmost picture. This intuition is confirmed by numerous experiments on real-world data from a broad variety of sources (Rissanen 1989; Vapnik 1998; Ripley 1996): if one naively fits a high-degree polynomial to a small sample (set of data points), then one obtains a very good fit to the data. Yet if one tests the inferred polynomial on a second set of data coming from the same source, it typically fits this test data very badly in the sense that there is a large distance between the polynomial and the new data points. We say that the polynomial overfits the data. Indeed, all model selection methods that are used in practice either implicitly or explicitly choose 

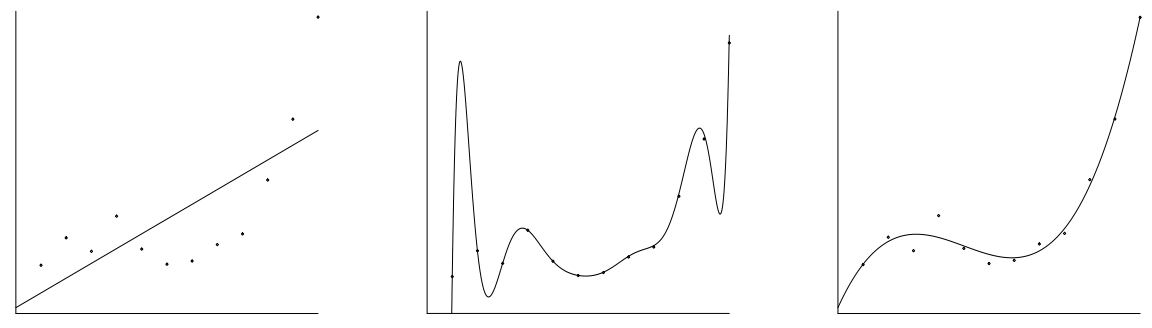

Figure 1.1 A simple, a complex and a tradeoff (third-degree) polynomial.

a tradeoff between goodness-of-fit and complexity of the models involved. In practice, such tradeoffs lead to much better predictions of test data than one would get by adopting the "simplest" (one degree) or most "complex"3 ( $n$-1-degree) polynomial. MDL provides one particular means of achieving such a tradeoff.

It will be useful to distinguish between "model", "model class" and "(point) hypothesis." This terminology is explained in the box on page 15, and will be discussed in more detail in Section 2.4, page 69. In our terminology, the problem described in Example 1.3 is a "point hypothesis selection problem" if we are interested in selecting both the degree of a polynomial and the corresponding parameters; it is a "model selection problem" if we are mainly interested in selecting the degree.

To apply MDL to polynomial or other types of hypothesis and model selection, we have to make precise the somewhat vague insight "learning may be viewed as data compression." This can be done in various ways. We first explain the earliest and simplest implementation of the idea. This is the socalled two-part code version of MDL:

3. Strictly speaking, in our context it is not very accurate to speak of "simple" or "complex" polynomials; instead we should call the set of first degree polynomials "simple," and the set of 100th-degree polynomials "complex." 


\section{Crude Two-Part Version of MDL Principle (Informally Stated)}

Let $\mathcal{H}_{1}, \mathcal{H}_{2}, \ldots$ be a list of candidate models (e.g., $\mathcal{H}_{\gamma}$ is the set of $\gamma$ th degree polynomials), each containing a set of point hypotheses (e.g., individual polynomials). The best point hypothesis $H \in \mathcal{H}=\mathcal{H}_{1} \cup \mathcal{H}_{2} \cup \ldots$ to explain the data $D$ is the one which minimizes the sum $L(H)+L(D \mid H)$, where

- $L(H)$ is the length, in bits, of the description of the hypothesis; and

- $L(D \mid H)$ is the length, in bits, of the description of the data when encoded with the help of the hypothesis.

The best model to explain $D$ is the smallest model containing the selected $H$.

The terminology "crude MDL" is explained in the next subsection. It is not standard, and it is introduced here for pedagogical reasons.

Example 1.4 [Polynomials, cont.] In our previous example, the candidate hypotheses were polynomials. We can describe a polynomial by describing its coefficients at a certain precision (number of bits per parameter). Thus, the higher the degree of a polynomial or the precision, the more bits we need to describe it and the more "complex" it becomes. A description of the data "with the help of" a hypothesis means that the better the hypothesis fits the data, the shorter the description will be. A hypothesis that fits the data well gives us a lot of information about the data. Such information can always be used to compress the data. Intuitively, this is because we only have to code the errors the hypothesis makes on the data rather than the full data. In our polynomial example, the better a polynomial $H$ fits $D$, the fewer bits we need to encode the discrepancies between the actual $y$-values $y_{i}$ and the predicted $y$-values $H\left(x_{i}\right)$. We can typically find a very complex point hypothesis (large $L(H)$ ) with a very good fit (small $L(D \mid H)$ ). We can also typically find a very simple point hypothesis (small $L(H)$ ) with a rather bad fit (large $L(D \mid H)$ ). The sum of the two description lengths will be minimized at a hypothesis that is quite (but not too) "simple," with a good (but not perfect) fit.

\subsubsection{From Crude to Refined MDL}

Crude MDL picks the $H$ minimizing the sum $L(H)+L(D \mid H)$. To make this procedure well defined, we need to agree on precise definitions for the 


\section{Models and Model Classes; (Point) Hypotheses}

We use the word model to refer to a set of probability distributions or functions of the same functional form. E.g., the "first-order Markov model" is the set of all probability distributions that are first-order Markov chains. The "model of $k$ th degree polynomials" is the set of all $k$ th degree polynomials for some fixed $k$.

We use the word model class to refer to a family (set) of models, e.g. "the model class of all polynomials" or "the model class of all Markov chains of each order." The definitions of "model" and "model class" are chosen so that they agree with how these words are used in statistical practice. Therefore they are intentionally left somewhat imprecise.

We use the word hypothesis to refer to an arbitrary set of probability distributions or functions. We use the word point hypothesis to refer to a single probability distribution (e.g. a Markov chain with all parameter values specified) or function (e.g. a particular polynomial). In parametric inference (Chapter 2), a point hypothesis corresponds to a particular parameter value. A point hypothesis may also be viewed as an instantiation of a model.

What we call "point hypothesis" is called "simple hypothesis" in the statistics literature; our use of the word "model (selection)" coincides with its use in much of the statistics literature; see Section 2.3, page 62 where we give several examples to clarify our terminology.

Figure 1.2 Models and Model Classes; (Point) Hypotheses.

codes (description methods) giving rise to lengths $L(D \mid H)$ and $L(H)$. We now discuss these codes in more detail. We will see that the definition of $L(H)$ is problematic, indicating that we somehow need to "refine" our crude MDL principle.

Definition of $L(D \mid H)$ Consider a two-part code as described above, and assume for the time being that all $H$ under consideration define probability distributions. If $H$ is a polynomial, we can turn it into a distribution by mak- 
ing the additional assumption that the $Y$-values are given by $Y=H(X)+Z$, where $Z$ is a normally distributed noise term with mean 0 .

For each $H$ we need to define a code with length $L(\cdot \mid H)$ such that $L(D \mid H)$ can be interpreted as "the codelength of $D$ when encoded with the help of $H . "$ It turns out that for probabilistic hypotheses, there is only one reasonable choice for this code; this is explained at length in Chapter 5. It it is the so-called Shannon-Fano code, satisfying, for all data sequences $D$, $L(D \mid H)=-\log P(D \mid H)$, where $P(D \mid H)$ is the probability mass or density of $D$ according to $H$. Such a code always exists, as we explain in Chapter 3, in the box on page 96.

Definition of $L(H)$ : A Problem for Crude MDL It is more problematic to find a good code for hypotheses $H$. Some authors have simply used "intuitively reasonable" codes in the past, but this is not satisfactory: since the description length $L(H)$ of any fixed point hypothesis $H$ can be very large under one code, but quite short under another, our procedure is in danger of becoming arbitrary. Instead, we need some additional principle for designing a code for $\mathcal{H}$.

In the first publications on MDL (Rissanen 1978; Rissanen 1983), it was implicitly advocated to choose some sort of minimax code for each $\mathcal{H}_{\gamma}$, minimizing the shortest worst-case total description length $L(H)+L(D \mid H)$, where the worst-case is over all possible data sequences. Thus, the MDL principle is employed at a "meta-level" to choose a code for $\mathcal{H}_{\gamma}$. This idea, already implicit in Rissanen's early work abut perhaps for the first time stated and formalized in a completely precise way Barron and Cover (1991), is the first step towards "refined" MDL.

More Problems for Crude MDL We can use crude MDL to code any sequence of data $D$ with a total description length $L(D):=\min _{H}\{L(D \mid H)+$ $L(H)\}$. But it turns out that this code is incomplete: one can show that there exist other codes $L^{\prime}$ which for some $D$ achieve strictly smaller codelength $\left(L^{\prime}(D)<L(D)\right)$, and for no $D$ achieve larger codelength (Chapter 6, Example 6.4). It seems strange that our "minimum description length" principle should be based on codes which are incomplete (inefficient) in this sense. Another, less fundamental problem with two-part codes is that, if designed in a minimax way as indicated above, they require a cumbersome discretization of the model space $\mathcal{H}$, which is not always feasible in practice. The final problem we mention is that, while it is clear how to use crude two-part codes for 
point hypothesis and model selection, it is not immediately clear how they can be used for prediction.

Later, Rissanen (1984) realized that these problems could be side-stepped by using one-part rather than two-part codes. As we explain below, it depends on the situation at hand whether a one-part or a two-part code should be used. Combining the idea of designing codes so as to achieve essentially minimax optimal codelengths with the combined use of one-part and twopart codes (whichever is appropriate for the situation at hand) has culminated in a theory of inductive inference that we call refined MDL. We discuss it in more detail in the next subsection.

\section{Crude Two-Part MDL (Part I, Chapter 5 of this book)}

In this book, we use the term "crude MDL" to refer to applications of MDL for model and hypothesis selection of the type described in the box on page 14, as long as the hypotheses $H \in \mathcal{H}$ are encoded in "intuitively reasonable" but ad-hoc ways.

Refined MDL is sometimes based on one-part codes, sometimes on twopart codes, and sometimes on a combination of these, but, in contrast to crude MDL, the codes are invariably designed according to some minimax principles. If there is a choice, one should always prefer refined MDL, but in some exotic modeling situations, the use of crude MDL is inevitable.

Part I of this book first discusses all probabilistic, statistical and information-theoretic preliminaries (Chapters 2-4) and culminates in a description of crude two-part MDL (Chapter 5). Refined MDL is described only in Part III.

\subsubsection{Universal Coding and Refined MDL}

In refined MDL, we associate a code for encoding $D$ not with a single $H \in \mathcal{H}$, but with the full model $\mathcal{H}$. Thus, given model $\mathcal{H}$, we encode data not in two parts but we design a single one-part code with lengths $\bar{L}(D \mid \mathcal{H})$. This code is designed such that whenever there is a member of (parameter in) $\mathcal{H}$ that fits the data well, in the sense that $L(D \mid H)$ is small, then the codelength $\bar{L}(D \mid \mathcal{H})$ will also be small. Codes with this property are called universal codes in the information-theoretic literature (Barron, Rissanen, and Yu 1998): 


\section{Universal Coding (Part II of This Book)}

There exist at least four types of universal codes:

1. The normalized maximum likelihood (NML) code and its variations.

2. The Bayesian mixture code and its variations.

3. The prequential plug-in code

4. The two-part code

These codes are all based on entirely different coding schemes, but in practice, lead to very similar codelengths $\bar{L}(D \mid \mathcal{H})$. Part II of this book is entirely devoted to universal coding. The four types of codes are introduced in Chapter 6. This is follows by a separate chapter for each code.

For each model $\mathcal{H}$, there are many different universal codes we can associate with $\mathcal{H}$. When applying MDL, we have a preference for the one that is minimax optimal in a sense made precise in Chapter 6 . For example, the set $\mathcal{H}_{3}$ of third-degree polynomials is associated with a code with lengths $\bar{L}\left(\cdot \mid \mathcal{H}_{3}\right)$ such that, the better the data $D$ are fit by the best-fitting third-degree polynomial, the shorter the codelength $\bar{L}(D \mid \mathcal{H}) . \bar{L}(D \mid \mathcal{H})$ is called the stochastic complexity of the data given the model.

Refined MDL is a general theory of inductive inference based on universal codes that are designed to be minimax, or close to minimax optimal. It has mostly been developed for model selection, estimation and prediction. To give a first flavor, we initially discuss model selection, where, arguably, it has the most new insights to offer:

\subsubsection{Refined MDL for Model Selection}

Parametric Complexity A fundamental concept of refined MDL for model selection is the parametric complexity of a parametric model $\mathcal{H}$ which we denote by $\operatorname{COMP}(\mathcal{H})$. This is a measure of the "richness" of model $\mathcal{H}$, indicating its ability to fit random data. This complexity is related to the number of degrees-of-freedom (parameters) in $\mathcal{H}$, but also to the geometrical structure of $\mathcal{H}$; see Example 1.5. To see how it relates to stochastic complexity, let, for given data $D, \hat{H}$ denote the distribution in $\mathcal{H}$ which maximizes the probability, and hence minimizes the codelength $L(D \mid \hat{H})$ of $D$. It turns out 
that

$$
\bar{L}(D \mid \mathcal{H})=\text { stochastic complexity of } D \text { given } \mathcal{H}=L(D \mid \hat{H})+\operatorname{COMP}(\mathcal{H}) .
$$

Refined MDL model selection between two parametric models $\mathcal{H}_{1}$ and $\mathcal{H}_{2}$ (such as the models of first and second degree polynomials) now proceeds as follows. We encode data $D$ in two stages. In the first stage, we encode a number $j \in\{1,2\}$. In the second stage, we encode the data using the universal code with lengths $\bar{L}\left(D \mid \mathcal{H}_{j}\right)$. As in the two-part code principle, we then select the $\mathcal{M}_{j}$ achieving the minimum total two-part codelength,

$$
\min _{j \in\{1,2\}}\left\{L(j)+\bar{L}\left(D \mid \mathcal{H}_{j}\right)\right\}=\min _{j \in\{1,2\}}\{L(j)+L(D \mid \hat{H})+\operatorname{COMP}(\mathcal{H})\} .
$$

Since the worst-case optimal code to encode $j$ needs only 1 bit to encode either $j=1$ or $j=2$, we use a code for the first-part such that $L(1)=L(2)=1$. But this means that $L(j)$ plays no role in the minimization, and we are effectively selecting the model such that the stochastic complexity of the given data $D$ is smallest. ${ }^{4}$ Thus, in the end we select the model minimizing the one-part codelength of the data. Nevertheless, refined MDL model selection involves a tradeoff between two terms: a goodness-of-fit term $L(D \mid \hat{H})$ and a complexity term $\operatorname{COMP}(\mathcal{H})$. However, because we do not explicitly encode hypotheses $H$ anymore, there is no potential for arbitrary codelengths anymore. The resulting procedure can be interpreted in several different ways, some of which provide us with rationales for MDL model selection beyond the pure coding interpretation (Chapter 14):

1. Counting/differential geometric interpretation The parametric complexity of a model is the logarithm of the number of essentially different, distinguishable point hypotheses within the model.

2. Two-part code interpretation For large samples, the stochastic complexity can be interpreted as a two-part codelength of the data after all, where hypotheses $H$ are encoded with a special code that works by first discretizing the model space $\mathcal{H}$ into a set of "maximally distinguishable hypotheses," and then assigning equal codelength to each of these.

3. Bayesian interpretation In many cases, refined MDL model selection coincides with Bayes factor model selection based on a noninformative prior such as Jeffreys' prior (Bernardo and Smith 1994).

4. The reason we include $L(j)$ at all in (1.4) is to maintain consistency with the case where we need to select between an infinite number of models. In that case, it is necessary to include $L(j)$. 
4. Prequential interpretation MDL model selection can be interpreted as selecting the model with the best predictive performance when sequentially predicting unseen test data, in the sense described in Chapter 6, Section 6.4 and Chapter 9. This makes it an instance of Dawid's (1984) prequential model validation and also relates it to cross-validation methods; see Chapter 17, Sections 17.5 and 17.6.

In Section 1.6.1 we show that refined MDL allows us to compare models of different functional form. It even accounts for the phenomenon that different models with the same number of parameters may not be equally "complex."

\subsubsection{General Refined MDL: Prediction and Hypothesis Selection}

Model selection is just one application of refined MDL. The two other main applications are point hypothesis selection and prediction. These applications can also be interpreted as methods for parametric and nonparametric estimation. In fact,it turns out that large parts of MDL theory can be reinterpreted as a theory about sequential prediction of future data given previously seen data. This "prequential" interpretation of MDL (Chapter 15) is at least as important as the coding interpretation. It is based on the fundamental correspondence between probability distributions and codes via the Shannon-Fano code that we alluded to before, when explaining the code with lengths $L(D \mid H)$; see the box on page 96 . This correspondence allows us to view any universal code $\bar{L}(\cdot \mid \mathcal{H})$ as a strategy for sequentially predicting data, such that the better $\mathcal{H}$ is suited as a model for the data, the better the predictions will be.

MDL prediction and hypothesis selection are mathematically cleaner than MDL model selection: in Chapter 15, we provide theorems (Theorem 15.1 and Theorem 15.3) which, in the respective contexts of prediction and hypothesis selection, express that, in full generality, good data compression implies fast learning, where "learning" is defined as "finding a hypothesis that is in some sense close to an imagined "true state of the world." There are similar theorems for model selection, but these lack some of the simplicity and elegance of Theorem 15.1 and Theorem 15.3.

Probabilistic vs. Nonprobabilistic MDL Like most other authors on MDL, in this book we confine ourselves to probabilistic hypotheses, also known as probabilistic sources. These are hypotheses that take the form of probability distributions over the space of possible data sequences. The examples we give in this chapter (Examples 1.3 and 1.5) involve hypotheses $H$ that are functions 
from some space $\mathcal{X}$ to another space $\mathcal{Y}$; at first sight, these are not "probabilistic." We will usually assume that for any given $x$, we have $y=H(x)+Z$ where $Z$ is a noise term with a known distribution. Typically, the noise $Z$ will be assumed to be Gaussian (normally) distributed. With such an additional assumption, we may view "functional" hypotheses $H: \mathcal{X} \rightarrow \mathcal{Y}$ as "probabilistic" after all. Such a technique of turning functions into probability distributions is customary in statistics, and we will use it throughout large parts of this book. Whenever we refer to MDL, we implicitly assume that we deal with probabilistic models. We should note though that there exists variations of MDL that directly work with universal codes relative to functional hypotheses such as polynomials (see Section 1.9.1, and Chapter 17, Section 17.10).

\section{Fixing Notation}

We use the symbol $H$ for general point hypotheses, that may either represent a probabilistic source or a deterministic function. We use $\mathcal{H}$ for sets of such general point hypotheses. We reserve the symbol $\mathcal{M}$ for probabilistic models and model classes. We denote probabilistic point hypotheses by $P$, and point hypotheses that are deterministic functions by $h$.

Individual-Sequence vs. Expectation-based MDL Refined MDL is based on minimax optimal universal codes. Broadly speaking, there are two different ways to define what we mean by minimax optimality. One is to look at the worst-case codelength over all possible sequences. We call this individualsequence MDL. An alternative is to look at expected codelength, where the expectation is taken over some probability distribution, usually but not always assumed to be a member of the model class $\mathcal{M}$ under consideration. We call this expectation-based MDL. We discuss the distinction in detail in Part III of the book; see also the box on page 407. The individual-sequence approach is the one taken by Rissanen, the main originator of MDL, and we will mostly follow it throughout this book.

The Luckiness Principle In the individual-sequence approach, the minimax optimal universal code is given by the normalized maximum likelihood (NML) code that we mentioned above. A problem is that for many (in fact, most) practically interesting models, the NML code is not well defined. In 
such cases, a minimax optimal code does not exist. As we explain in Chapter 11, in some cases one can get around this problem using so-called "conditional NML" codes, but in general, one needs to use codes based on a modified minimax principle, which we call the luckiness principle. Although it has been implicitly used in MDL since its inception, I am the first to use the term "luckiness principle" in an MDL context; see the box on page 92, Chapter 3; the developments in Chapter 11, Section 11.3, where we introduce the concept of a luckiness function; and the discussion in Chapter 17, Section 17.2.1.

The luckiness principle reintroduces some subjectivity in MDL code design. This seems to bring us back to the ad-hoc codes used in crude two-part MDL. The difference however is that with luckiness functions, we can precisely quantify the effects of this subjectivity: for each possible data sample $D$ that we may observe, we can indicate how "lucky" we are on the sample, i.e. how many extra bits we need compared to encode $D$ compared to the best hypothesis that we have available for $D$. This idea significantly extends the applicability of refined MDL methods.

MDL is a Principle Contrary to what is often thought, MDL, and even, "modern, refined MDL" is not a unique, single method of inductive inference. Rather, it represents a general principle for doing inductive inference. The principle may (and will) be formulated precisely enough to allow us to establish, for many given methods (procedures, learning algorithms) "this method is an instance of MDL" or "this is not an instance of MDL. But nevertheless:

\footnotetext{
MDL Is a Principle, Not a Unique Method

Being a principle, MDL gives rise to several methods of inductive inference. There is no single "uniquely optimal MDL method/procedure/algorithm." Nevertheless, in some special situations (e.g. simple parametric statistical models), one can clearly distinguish between good and not so good versions of MDL, and something close to "an optimal MDL method" exists.
} 


\section{Summary: Refined MDL (Part III of This Book)}

Refined MDL is a method of inductive inference based on universal codes which are designed to have some minimax optimality properties. Each model $\mathcal{H}$ under consideration is associated with a corresponding universal code. In this book we restrict ourselves to probabilistic $\mathcal{H}$. Refined MDL has mainly been developed for model selection, point hypothesis selection and prediction.

Refined MDL comes in two versions: individual-sequence and expectation-based refined MDL, depending on whether the universal codes are designed to be optimal in an individual-sequence or in an expected sense. If the minimax optimal code relative to a model $\mathcal{M}$ is not defined, some element of subjectivity is introduced into the coding using a luckiness function. A more precise overview is given in the box on page 406.

In the remainder of this chapter we will mostly concentrate on MDL for model selection.

\subsection{Some Remarks on Model Selection}

Model selection is a controversial topic in statistics. Although most people agree that it is important, many say it can only be done on external grounds, and never by merely looking at the data. Still, a plethora of automatic model selection methods has been suggested in the literature. These can give wildly different results on the same data, one of the main reasons being that they have often been designed with different goals in mind. This section starts with a further example that motivates the need for model selection, and it then discusses several goals that one may have in mind when doing model selection. These issues are discussed in a lot more detail in Chapter 14. See also Chapter 17, especially Section 17.3, where we compare MDL model selection to the standard model selection methods AIC and BIC.

\subsubsection{Model Selection among Non-Nested Models}

Model selection is often used in the following context: two researchers or research groups $A$ and $B$ propose entirely different models $\mathcal{M}_{A}$ and $\mathcal{M}_{B}$ as an explanation for the same data $D$. This situation occurs all the time in applied sciences like econometrics, biology, experimental psychology, etc. For 
example, group $A$ may have some general theory about the phenomenon at hand which prescribes that the trend in data $D$ is given by some polynomial. Group $B$ may think that the trend is better described by some neural network; a concrete case will be given in Example 1.3 below. $A$ and $B$ would like to have some way of deciding which of their two models is better suited for the data at hand. If they simply decide on the model containing the hypothesis (parameter instantiation) that best fits the data, they once again run the risk of overfitting: if model $\mathcal{M}_{A}$ has more degrees of freedom (parameters) than model $\mathcal{M}_{B}$, it will typically be able to better fit random noise in the data. It may then be selected even if $\mathcal{M}_{B}$ actually better captures the underlying trend (regularity) in the data. Therefore, just as in the hypothesis selection example, deciding whether $\mathcal{M}_{A}$ or $\mathcal{M}_{B}$ is a better explanation for the data should somehow depend on how well $\mathcal{M}_{A}$ and $\mathcal{M}_{B}$ fit the data and on the respective "complexities" of $\mathcal{M}_{A}$ and $\mathcal{M}_{B}$.

In the polynomial case discussed before, there was a countably infinite number of "nested" $\mathcal{M}_{\gamma}$ (i.e. $\mathcal{M}_{\gamma} \subset \mathcal{M}_{\gamma+1}$ ). In contrast, we now deal with a finite number of entirely unrelated models $\mathcal{M}_{\gamma}$. But there is nothing that stops us from using MDL model selection as "defined" above.

\section{Example 1.5 [Selecting Between Models of Different Functional Form]}

Consider two models from psychophysics describing the relationship between physical dimensions (e.g., light intensity) and their psychological counterparts (e.g. brightness) (Myung, Balasubramanian, and Pitt 2000): $y=$ $a x^{b}+Z$ (Stevens's model) and $y=a \ln (x+b)+Z$ (Fechner's model) where $Z$ is a normally distributed noise term. Both models have two free parameters; nevertheless, according to the refined version of MDL model selection to be introduced in Part III, Chapter 14 of this book, Stevens's model is in a sense "more complex" than Fechner's (see page 417). Roughly speaking, this means there are a lot more data patterns that can be explained by Stevens's model than can be explained by Fechner's model. Somewhat more precisely, the number of data patterns (sequences of data) of a given length that can be fit well by Stevens's model is much larger than the number of data patterns of the same length that can be fit well by Fechner's model. Therefore, using Stevens's model we run a larger risk of "overfitting."

In the example above, the goal was to select between a power law and a logarithmic relationship. In general, we may of course come across model selection problems involving neural networks, polynomials, Fourier or wavelet expansions, exponential functions - anything may be proposed and tested. 
could have tried to learn $g$ using a model class $\mathcal{H}$ containing the function $y=\exp (x)$. But in general, both our imagination and our computational resources are limited, and we may be forced to use imperfect models.

If, based on a small sample, we choose the best-fitting polynomial $\hat{h}$ within the set of all polynomials, then, even though $\hat{h}$ will fit the data very well, it is likely to be quite unrelated to the "true" $g$, and $\hat{h}$ may lead to disastrous predictions of future data. The reason is that, for small samples, the set of all polynomials is very large compared to the set of possible data patterns that we might have observed. Therefore, any particular data pattern can only give us very limited information about which high-degree polynomial best approximates $g$. On the other hand, if we choose the best-fitting $\hat{h}^{\circ}$ in some much smaller set such as the set of second-degree polynomials, then it is highly probable that the prediction quality (mean squared error) of $\hat{h}^{\circ}$ on future data is about the same as its mean squared error on the data we observed: the size (complexity) of the contemplated model is relatively small compared to the set of possible data patterns that we might have observed. Therefore, the particular pattern that we do observe gives us a lot of information on what second-degree polynomial best approximates $g$.

Thus, (a) $\hat{h}^{\circ}$ typically leads to better predictions of future data than $\hat{h}$; and (b) unlike $\hat{h}, \hat{h}^{\circ}$ is reliable in that it gives a correct impression of how good it will predict future data even if the "true" $g$ is "infinitely" complex. This idea does not just appear in MDL, but is also the basis of the structural risk minimization approach (Vapnik 1998) and many standard statistical methods for nonparametric inference; see Chapter 17, Section 17.10. In such approaches one acknowledges that the data-generating machinery can be infinitely complex (e.g., not describable by a finite degree polynomial). Nevertheless, it is still a good strategy to approximate it by simple hypotheses (low-degree polynomials) as long as the sample size is small. Summarizing:

\section{The Inherent Difference between Under- and Overfitting}

If we choose an overly simple model for our data, then the best-fitting point hypothesis within the model is likely to be almost the best predictor, within the simple model, of future data coming from the same source. If we overfit (choose a very complex model) and there is noise in our data, then, even if the complex model contains the "true" point hypothesis, the best-fitting point hypothesis within the model may lead to very bad predictions of future data coming from the same source. 
This statement is very imprecise and is meant more to convey the general idea than to be completely true. The fundamental consistency theorems for MDL prediction and hypothesis selection (Chapter 15, Theorem 15.1 and Theorem 15.3), as well as their extension to model selection (Chapter 16), are essentially just variations of this statement that are provably true.

The Future and The Past Our analysis depends on the data items $\left(x_{i}, y_{i}\right)$ to be probabilistically independent. While this assumption may be substantially weakened, we can justify the use of MDL and other forms of Occam's razor only if we are willing to adopt some (possibly very weak) assumption of the sort "training data and future data are from the same source": future data should (at least with high probability) be subject to some of the same regularities as training data. Otherwise, $D$ and $D^{\prime}$ may be completely unrelated and no method of inductive inference can be expected to work well. This is indirectly related to the grue-paradox (Goodman 1955).

\section{MDL and Occam's Razor}

While MDL does have a built-in preference for selecting "simple" models (with small "parametric complexity"), this does not at all mean that applying MDL only makes sense in situations where simpler models are more likely to be true. MDL is a methodology for inferring models from data, not a statement about how the world works! For small sample sizes, it prefers simple models. It does so not because these are "more likely to be true" (they often are not). Instead, it does so because this tends to select the model that leads to the best predictions of future data from the same source. For small sample sizes this may be a model much simpler than the model containing the "truth" (assuming for the time being that such a model containing the "truth" exists in the first place).

In fact, some of MDL's most useful and successful applications are in nonparametric statistics where the "truth" underlying data is typically assumed to be "infinitely" complex (see Chapter 13 and Chapter 15). 


\subsection{History and Forms of MDL}

The practical MDL principle that we discuss in this book has mainly been developed by J. Rissanen in a series of papers starting with (Rissanen 1978). It has its roots in the theory of Kolmogorov complexity (Li and Vitányi 1997), developed in the 1960s by Solomonoff (1964), Kolmogorov (1965) and Chaitin $(1966,1969)$. Among these authors, Solomonoff (a former student of the famous philosopher of science, Rudolf Carnap) was explicitly interested in inductive inference. The 1964 paper contains explicit suggestions on how the underlying ideas could be made practical, thereby foreshadowing some of the later work on two-part MDL. While Rissanen was not aware of Solomonoff's work at the time, Kolmogorov's [1965] paper did serve as an inspiration for Rissanen's (1978) development of MDL. Still, Rissanen's practical MDL is quite different from the idealized forms of MDL that have been directly based on Kolmogorov complexity, which we discussed in Section 1.4.

Another important inspiration for Rissanen was Akaike's AIC method for model selection (Chapter 17, Section 17.3), essentially the first model selection method based on information-theoretic ideas (Akaike 1973). Even though Rissanen was inspired by AIC, both the actual method and the underlying philosophy are substantially different from MDL.

Minimum Message Length MDL is much closer related to the Minimum Message Length (MML) Principle (Wallace 2005), developed by Wallace and his coworkers in a series of papers starting with the groundbreaking (Wallace and Boulton 1968); other milestones are (Wallace and Boulton 1975) and (Wallace and Freeman 1987). Remarkably, Wallace developed his ideas without being aware of the notion of Kolmogorov complexity. Although Rissanen became aware of Wallace's work before the publication of (Rissanen 1978), he developed his ideas mostly independently, being influenced rather by Akaike and Kolmogorov. Indeed, despite the close resemblance of both methods in practice, the underlying philosophy is very different - see Chapter 17 , Section 17.4.

Refined MDL The first publications on MDL only mention two-part codes. Important progress was made by Rissanen (1984), in which prequential codes are employed for the first time and Rissanen (1987), who introduced the Bayesian mixture codes into MDL. This led to the development of the notion

of stochastic complexity as the shortest codelength of the data given a model 
(Rissanen 1986c; Rissanen 1987). However, the connection to Shtarkov's normalized maximum likelihood code was not made until 1996, and this prevented the full development of the notion of "parametric complexity." In the mean time, in his impressive Ph.D. thesis, Barron (1985) showed how a specific version of the two-part code criterion has excellent frequentist statistical consistency properties. This was extended by Barron and Cover (1991) who achieved a breakthrough for two-part codes: they gave clear prescriptions on how to design codes for hypotheses, relating codes with good minimax codelength properties to rates of convergence in statistical consistency theorems. Some of the ideas of Rissanen (1987) and Barron and Cover (1991) were, as it were, unified when Rissanen (1996) introduced the normalized maximum likelihood code. The resulting theory was summarized for the first time by Barron, Rissanen, and Yu (1998), and is the subject of this book. Whenever we need to distinguish it from other forms of MDL, we call it "refined MDL."

\subsubsection{What Is MDL?}

"MDL" is used by different authors in somewhat different meanings, and it may be useful to review these. Some authors use MDL as a broad umbrella term for all types of inductive inference based on finding a short codelength for the data. This would, for example, include the "idealized" versions of MDL based on Kolmogorov complexity (page 11) and Wallaces's MML principle (see above). Some authors take an even broader view and include all inductive inference that is based on data compression, even if it cannot be directly interpreted in terms of codelength minimization. This includes, for example the work on similarity analysis and clustering based on the normalized compression distance (Cilibrasi and Vitányi 2005).

On the other extreme, for historical reasons, some authors use the $M D L$ Criterion to describe a very specific (and often not very successful) model selection criterion equivalent to BIC (see Chapter 17, Section 17.3).

As already indicated, we adopt the meaning of the term that is embraced in the survey (Barron, Rissanen, and Yu 1998), written by arguably the three most important contributors to the field: we use MDL for general inference based on universal models. Although we concentrate on hypothesis selection, model selection and prediction, this idea can be further extended to many other types of inductive inference. These include denoising (Rissanen 2000; Hansen and Yu 2000; Roos, Myllymäki, and Tirri 2005), similarity analysis and clustering (Kontkanen, Myllymäki, Buntine, Rissanen, and Tirri 2005), outlier detection and transduction (as defined in (Vapnik 1998)), and many others. In 
such areas there has been less research and a "definitive" universal-model based MDL approach has not yet been formulated. We do expect, however, that such research will take place in the future: one of the main strengths of "MDL" in this broad sense is that it can be applied to ever more exotic modeling situations, in which the models do not resemble anything that is usually encountered in statistical practice. An example is the model of context-free grammars, already considered by Solomonoff (1964).

Another application of universal-model based MDL is the type of problem usually studied in statistical learning theory (Vapnik 1998); see also Chapter 17, Section 17.10. Here the goal is to directly learn functions (such as polynomials) to predict $Y$ given $X$, without making any specific probabilistic assumptions about the noise. MDL has been developed in some detail for such problems, most notably classification problems, where $Y$ takes its values in a finite set - spam filtering is a prototypical example; here $X$ stands for an email message, and $Y$ encodes whether or not it is spam. An example is the application of MDL to decision tree learning (Quinlan and Rivest 1989; Wallace and Patrick 1993; Mehta, Rissanen, and Agrawal 1995). Some MDL theory for such cases has been developed (Meir and Merhav 1995; Yamanishi 1998; Grünwald 1998), but the existing MDL methods in this area can behave suboptimally. This is explained in Chapter 17, Section 17.10.2. Although we certainly consider it a part of "refined" MDL, we do not consider this "nonprobabilistic" MDL further in this book, except in Section 17.10.2.

\subsubsection{Literature}

Theoretical Contributions There have been numerous contributors to refined MDL theory, but there are three researchers that I should mention explicitly: J. Rissanen, B. Yu and A. Barron, who jointly wrote (Barron, Rissanen, and Yu 1998). For example, most of the results that connect MDL to traditional statistics (including Theorem 15.1 and Theorem 15.3 in Chapter 15) are due to A. Barron. This book contains numerous references to their work.

There is a close connection between MDL theory and work in universal coding ((Merhav and Feder 1998); see also Chapter 6) and universal prediction ((Cesa-Bianchi and Lugosi 2006); see also Chapter 17, Section 17.9).

Practical Contributions There have been numerous practical applications of MDL. The only three applications we describe in detail are a crude MDL method for learning Markov chains (Chapter 5); a refined MDL method for 
learning densities based on histograms (Chapter 13 and Chapter 15); and MDL regression (Chapter 12 and Chapter 14). Below we give a few representative examples of other applications and experimental results that have appeared in the literature. We warn the reader that this list is by no means complete! Hansen and Yu (2001) apply MDL to a variety of practical problems involving regression, clustering analysis, and time series analysis. In (Tabus, Rissanen, and Astola 2002; Tabus, Rissanen, and Astola 2003), MDL is used for classification problems arising in genomics. Lee (2002a,b) describes additive clustering with MDL. use MDL for image denoising and apply MDL to decision tree learning. use MDL for sequential prediction. In (Myung, Pitt, Zhang, and Balasubramanian 2000; Myung, Balasubramanian, and Pitt 2000), MDL is applied to a variety of model selection problems arising in cognitive psychology. All these authors apply modern, "refined" versions of MDL. Some references to older work, in which "crude" (but often quite sensible) ad-hoc codes are used, are (Friedman, Geiger, and Goldszmidt 1997; Allen and Greiner 2000; Allen, Madani, and Greiner 2003; Rissanen and Ristad 1994; Quinlan and Rivest 1989; Nowak and Figueiredo 2000; Liu and Moulin 1998; Ndili, Nowak, and Figueiredo 2001; Figueiredo, J. Leitão, and A.K.Jain 2000; Gao and Li 1989). In these papers, MDL is applied to learning Bayesian networks, grammar inference and language acquisition, learning decision trees, analysis of Poisson point processes (for biomedical imaging applications), image denoising, image segmentation, contour estimation, and Chinese handwritten character recognition respectively. MDL has also been extensively studied in time-series analysis, both in theory (Hannan and Rissanen 1982; Gerenscér 1987; Wax 1988; Hannan, McDougall, and Poskitt 1989; Hemerly and Davis 1989b; Hemerly and Davis 1989a; Gerencsér 1994) and practice (Wei 1992; Wagenmakers, Grünwald, and Steyvers 2006).

Finally, we should note that there have been a number of applications, especially in natural language learning, which, although practically viable, have been primarily inspired by "idealized MDL" and Kolmogorov complexity, rather than by the Rissanen-Barron-Yu style of MDL that we consider here. These include (Adriaans and Jacobs 2006; Osborne 1999; Starkie 2001) and my own (Grünwald 1996).

Other Tutorials, Introductions and Overviews The reader who prefers a shorter introduction to MDL than the present one may want to have a look at (Barron, Rissanen, and Yu 1998) (very theoretical and very comprehensive; presumes knowledge of information theory), (Hansen and Yu 2001) 
(presumes knowledge of statistics; describes several practical applications), (Lanterman 2001) (about comparing MDL, MML and asymptotic Bayesian approaches to model selection), or perhaps my own (Grünwald 2005), which is part of (Grünwald, Myung, and Pitt 2005), a "source book" for MDL theory and applications that contains chapters by most of the main contributors to the field.

Rissanen $(1989,2007)$ has written two books on MDL. While outdated as an introduction to MDL, the "little green book" (Rissanen 1989) is still very much worth reading for its clear exposition of the philosophy underlying MDL. (Rissanen 2007) contains a brief general introduction and then focuses on some recent research of Rissanen's, applying the renormalized maximum likelihood (RNML) distribution (Chapter 11) in regression and denoising, and formalizing the connection between MDL and Kolmogorov's structure function. In contrast to myself, Rissanen writes in accord with his own principle: while containing a lot of information, both texts are quite short.

\subsection{Summary and Outlook}

We have discussed the relationship between compression, regularity, and learning. We have given a first idea of what the MDL principle is all about, and of the kind of problems we can apply it to. In the next chapters, we present the mathematical background needed to describe such applications in detail. 